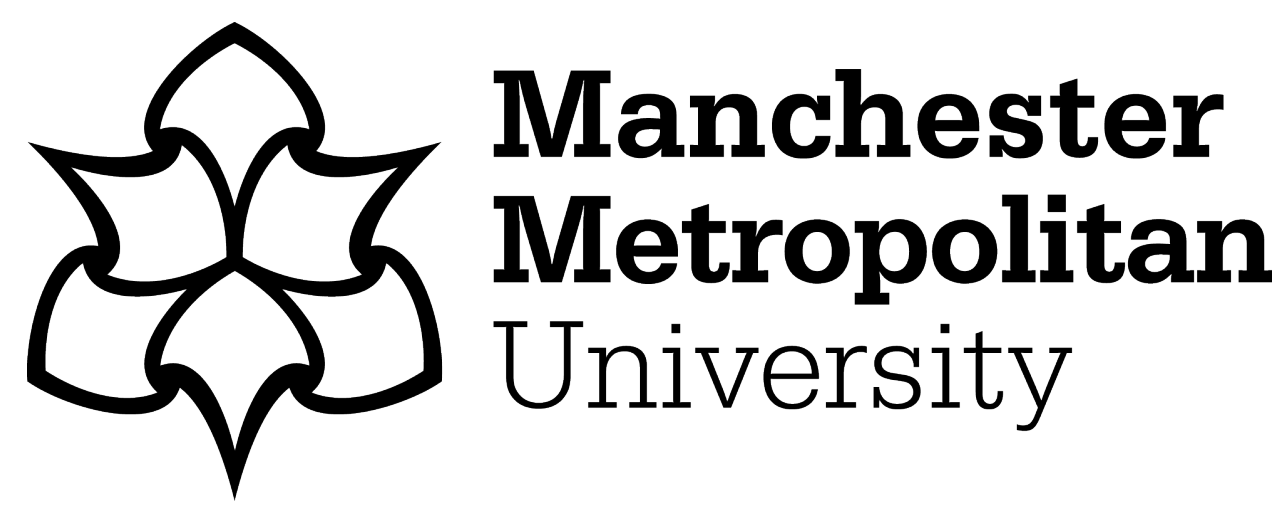

Sutcliffe, OB (2014) Classical and microwave-assisted synthesis of cucurbit[n]urils, hemicucurbit[n]urils and bambus[n]urils. In: Microwaves in Drug Discovery and Development Recent Advances. Future Medicine Ltd. ISBN 191041929X

Downloaded from: https://e-space.mmu.ac.uk/619414/

Publisher: Future Medicine Ltd

DOI: https://doi.org/10.4155/FSEB2013.13.88

Please cite the published version 


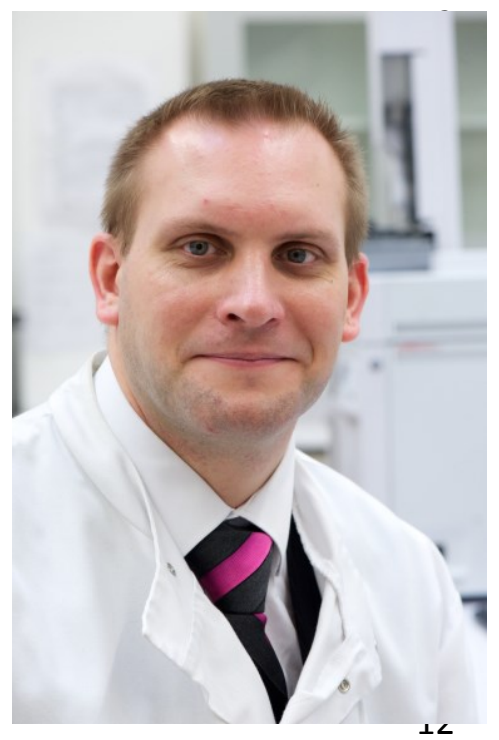

Oliver Sutcliffe graduated from the University of Liverpool with a BSc (Hons) in Chemical Sciences (1996) and subsequently obtained a PhD in Synthetic Organic Chemistry working on "New Extended Dipolar Systems" under Drs Richard C. Storr and Thomas L. Gilchrist from the same institution. In 1999, he joined Professor Martin R. Bryce's group at the University of Durham as a postdoctoral fellow investigating the application of enantiopure ferrocene ligands in asymmetric synthesis, before moving to industry and working as a medicinal chemist on the design, development and optimisation of drug molecules for a broad-range of biological targets, including cancer. He moved to the University of Strathclyde in 2006, obtained an MSc in Pharmaceutical Analysis and after a period at the European Directorate for the Quality of Medicines and Healthcare (EDQM) in

Strasbourg was appointed as a lecturer in the Strathclyde Institute of Pharmacy and Biomedical Sciences (SIPBS). In January 2012, Dr Sutcliffe joined the staff at Manchester Metropolitan University as both a Senior Lecturer in Pharmaceutical and Analytical Chemistry and Co-Director of the Manchester Centre for the Study of Legal Highs (MCSLH). Dr Sutcliffe's research interests include the isolation, identification and analysis of "new psychoactive substances" (formally known as "legal highs") such as mephedrone and its derivatives, and the development of simple, rapid and selective presumptive tests and devices which can be applied by "non-specialists" for the detection of illicit substances in the field. He is the co-inventor of 10 international patents and co-author of 33 peer-reviewed research articles.

\section{Author Contact Details}

Dr. Oliver B. Sutcliffe

School of Science and the Environment,

27 Manchester Metropolitan University,

28 John Dalton Building,

29 Chester Street,

Manchester. M1 5GD

Tel. +44 (0)161 2471531 
34 Cucurbit[n]urils are supramolecular structures made of glycoluril monomers linked by methylene bridges. The oxygen atoms are located along the peripheral edge of the macrocycle and are tilted inwards, forming a partly enclosed hydrophobic cavity that has been shown to bind to a range of organic molecules and inorganic ions. The name is derived from the resemblance of this molecule with a pumpkin of the family of Cucurbitaceae.

Key term 2: Hemicucurbit[n]uril

Hemicucurbit[n]urils are supramolecular structures made of $N$-substituted ethyleneureas monomers linked by methylene bridges. These macrocycles resemble a cucurbit[n]uril bisected along its equator where the ethyleneurea subunits adopt an alternating "zigzag" conformation. Unlike cucurbit[n]urils, hemicucurbit[n]urils have a limited ability to form inclusion complexes with organic compounds and do not to bind to inorganic ions.

Key term 3: Bambus[n]uril

Bambus[n]urils are supramolecular structures made of 2,4-disubstituted glycoluril monomers linked by methylene bridges. These macrocycles combines the features of both cucurbit[n]urils and hemicucurbit[n]urils to give a hyperbolic three-dimensional structure where the glycouril subunits adopt an alternating "zigzag" conformation and allow the formation of inclusion complexes. The name is derived from the resemblance of this molecule with the bamboo plant stem of the family of Bambusoidea.

\section{Key term 4: Microwave Synthesis}

Microwave synthesis is a rapidly expanding field in both organic and inorganic chemistries where microwave energy is focussed directly (and efficiently) into the reactive components of a mixture, rather than being transferred from the bulk solvent by convection/conduction (as occurs in conventionally heated reactions). This technique has been applied to a wide-range of chemical processes, using both solution- and solid-phase reagents, and is able to greatly enhance the rate and overall yield of many reactions leading to products, which in general, require minimal work-up and purification. 


\title{
Classical and microwave-assisted synthesis of cucurbit[n]urils,
} hemicucurbit[n]urils and bambus[n]urils

\author{
Oliver B. Sutcliffe
}

School of Science and the Environment, Manchester Metropolitan University, Chester Street, Manchester, UK. M1 5GD

\section{Abstract}

This chapter gives a concise overview of the synthetic and supramolecular chemistry of cucurbit[ $n]$ uril (CB $[n])$ family of macrocycles and expands it to hemicucurbit[n]urils (HemCB $[n])$ and bambus $[n]$ urils $(\mathrm{BU}[n])$. The application of microwave-assisted synthesis to access these macrocycles is reviewed. Several extensive papers describing the synthesis, physical and recognition properties and applications of $\mathrm{CB}[n] \mathrm{s}$ have been published and the reader is directed to these key sources throughout this chapter.

\section{Introduction}

The toroidal cucurbit[n]uril ( $\mathrm{CB}[n] ; n=5,6,7,8$ or 10 ) (1) family of macrocycles represent a unique class of compounds that display a variety of host-guest interactions [1 - 7]. Their symmetrical, oxygen-lined portals give access to a hydrophobic cavity that has been shown to bind organic or inorganic compounds, gases and alkali earth metal salts with binding constants of up to $10^{15} \mathrm{M}^{-1}$. Hemicucurbit[ $n$ ] urils (2) and bambus[n]urils (3) can be viewed as structurally-related to cucurbit[n]urils where the former are bisected along their equators and adopt a "zigzag" conformation [1], whilst the latter combines the structural features of both cucurbit[n]urils and hemicucurbit[n]urils to give a hyperbolic three-dimensional arrangement [8] (Figure 1). Cucurbit[n]urils have been extensively reviewed in terms of their synthesis, properties and potential applications in both chemical and pharmaceutical sciences and the author directs the reader to the recent series of articles published in the Israel Journal of Chemistry for more indepth information regarding selected aspects of $\mathrm{CB}[n]$ chemistry [7]. This chapter seeks to provide a concise overview of these macrocycles, their synthesis and applications and how the field has expanded to the structurallyrelated hemicucurbit[n]uril and bambus[ $n]$ uril families. 


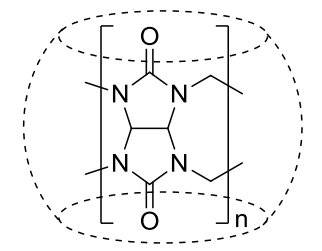

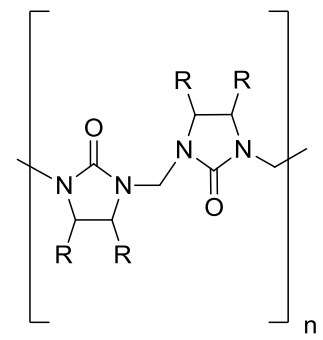

2

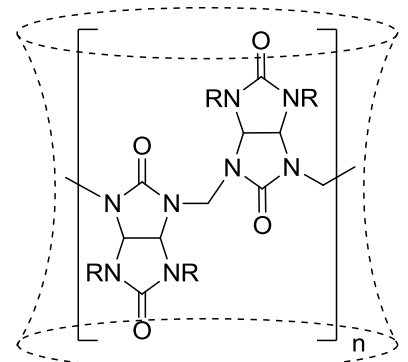

3

Figure 1. Structures of cucurbit[n]uril (1), hemicucurbit[n]uril (2) and bambus[ $n]$ uril (3)

\section{Synthesis of cucurbit[n]urils, hemicucurbit[n]urils and bambus[n]urils}

91 The large-scale production of $\mathrm{CB}[n] \mathrm{s}$ for commercial and industrial applications, particularly drug delivery, requires

92 reliable methods of synthesis that are effective in terms of both the ready availability of cheap starting materials and 93 energy consumption. Cucurbit[n]urils (6) are typically made through the condensation of glycoluril $(5, R=H)$ and 94 formaldehyde (or paraformaldehyde) in hot concentrated mineral acids (Scheme 1, Method A) [1 - 7, 9]. By changing 95 the type of acid used, the molarity of the acid and/or the concentration of glycoluril, the distribution of the $\mathrm{CB}[n]$ 96 products can be adjusted. For instance, synthesis in concentrated sulfuric acid typically produces samples containing $9788 \% \mathrm{CB}[6]$ with approximately $12 \% \mathrm{CB}[5]$ and inconsequential amounts of $\mathrm{CB}[7]$ and $\mathrm{CB}[8]$ [9]. Alternatively, the use 98 of $5 \mathrm{M}$ hydrochloric acid produces mixtures containing up to $35 \% \mathrm{CB}[7]$ through the use of very high concentrations 99 of glycoluril (circa. $1700 \mathrm{mg} \mathrm{mL}^{-1}$ ) and concentrated hydrochloric acid solutions produce mixtures containing up to $8 \%$ 100 $\mathrm{CB}[8][9]$. 


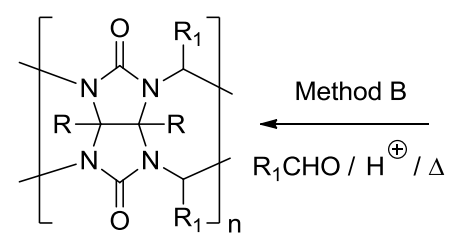

4

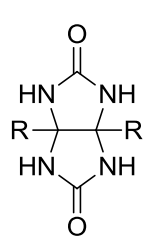

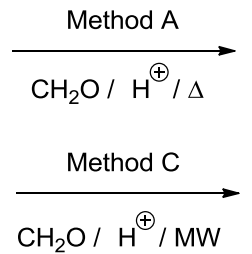

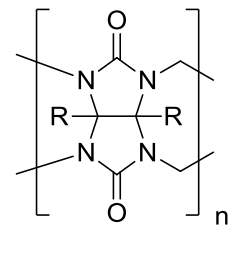

6

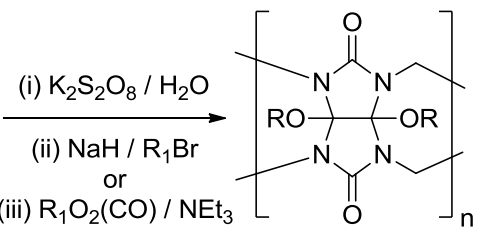

7

Scheme 1. Synthesis of cucurbit[n]urils $(n=5,6,7,8$ or 10$)$ and functionalised cucurbit $[n]$ urils $(n=5,6$ or 7$)$. Method $\mathrm{A}(n=5-10[\mathrm{R}=\mathrm{H}) ; n=5$ or $6[\mathrm{R}=$ Me or cyclohexyl- $])$; Method $\mathrm{B}\left(n=5-10, \mathrm{R}=\mathrm{R}_{1}=\mathrm{H}\right)$; Method C ( $n=5-10, \mathrm{R}=\mathrm{H}$, see Scheme 6 for conditions).

Efforts to further extend the supramolecular chemistry and improve the solubility of $\mathrm{CB}[n]$ in common solvents led to the development of efficient synthetic methods to prepare functionalized $\mathrm{CB}[n]$ derivatives. Principally three routes were envisaged: (i) condensation of glycoluril $(5, R=H)$ with substituted aldehydes to introduce substituents at the methylene bridgehead (4, Scheme 1, Method B); (ii) acid-catalysed condensation of substituted glycolurils $(\mathbf{5}, \mathrm{R}=$ alkyl, aryl etc.) with formaldehyde (Scheme 1, Method A) and finally (iii) direct functionalization of cucurbit[n]uril itself (for example: $6 \rightarrow 7$ ) (Scheme 1). The first route was unsuccessful and the second has met with little success with only $\mathrm{Me}_{10} \mathrm{CB}[5](6, n=5, \mathrm{R}=\mathrm{Me}), \mathrm{Me}_{10} \mathrm{CB}[6](6, n=6, \mathrm{R}=\mathrm{Me})$ and the cyclohexano-derivatives $(6, n=5$ or $6, \mathrm{R}=$ cyclohexano-) reported via this route [1]. CB[n] ( $n=6$ or 7) analogues utilising bis(phthalylhydrazide) and substituted glycoluril(bis-cyclic ether) as the building blocks have also been reported and show significant improvements in solubility in organic and aqueous media depending on the substituents present and have a similar binding capacity to $\mathrm{CB}[6][1]$.

Partially substituted $\mathrm{CB}[n]$ derivative, $\mathrm{Ph}{ }_{2} \mathrm{CB}[6](9 \mathrm{a}, \mathrm{R}=\mathrm{Ph})$ was first prepared by Nakamura et al. by sulphuric acidcatalysed mixed condensation of formaldehyde with diphenylglycoluril $(\mathbf{5}, \mathrm{R}=\mathrm{Ph})$ and glycoluril in a 1:5 ratio $[1,3]$ with Kim et al. patenting the synthesis, via di(o-nitro)phenylCB[6] (9b, $\left.\mathrm{R}=o-\mathrm{NO}_{2} \mathrm{Ph}\right)$ and di(p-nitro)phenylCB[6] (9c, $\mathrm{R}$ $\left.=p-\mathrm{NO}_{2} \mathrm{Ph}-\right)$, of di (o-amino)phenylCB [6] $\left(\mathbf{1 0 a}, \mathrm{R}=o-\mathrm{NH}_{2} \mathrm{Ph}\right)$ and di $\left(p\right.$-amino)phenylCB $[6]\left(\mathbf{1 0 b}, \mathrm{R}=p-\mathrm{NH}_{2} \mathrm{Ph}-\right)$ shortly thereafter [3] (Scheme 2). Other partially substituted derivatives, namely $\mathrm{Me}_{4} \mathrm{CB}[6]$ and $\left(\mathrm{Me}_{2} \mathrm{CyP}\right)_{n} \mathrm{CB}[6]$ (cyclopentano, CyP), have been reported $[1,3]$. 


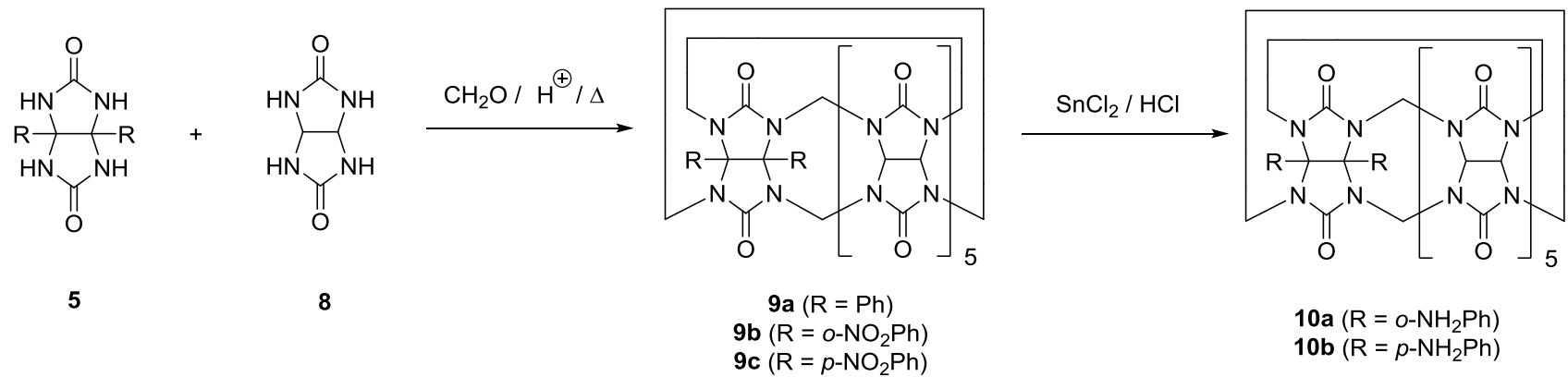

Scheme 2. Synthesis of functionalised cucurbit[n]urils.

A major step in the field was accomplished by the Kim et al. direct perhydroxylation of $\mathrm{CB}[n]$ using aqueous $\mathrm{K}_{2} \mathrm{~S}_{2} \mathrm{O}_{8}$ as an oxidant to provide the multi-functionalised derivative, $(\mathrm{HO})_{2 n} \mathrm{CB}[n](7, n=5,6$ or $7, \mathrm{R}=\mathrm{H})$, in low to moderate yield (5-45\% yield) (Scheme 1). Subsequent functionalization with either an alkyl bromide or anhydride yielded the corresponding alkoxy $\left(7, R=R_{1}\right)$ or acyloxy- $\left(7, R=C_{1} R_{1}\right)$ products which have exploited in numerous biological applications $[3,10]$. Scherman et al. refined the persulfate oxidation of $\mathrm{CB}[6]$ facilitating isolation of $(\mathrm{HO}){ }_{1} \mathrm{CB}[6](\mathbf{1 1})$ in $12 \%$ yield (Scheme 3). Concomitant transformation into the propargyloxyCB[6] (12) facilitates Huisgen azideacetylene click reaction to generate the self-complexing $\mathrm{CB}[6]$ derivative (13) [11]. Isaacs et al. who recently reported the gram scale synthesis of a number of monofunctionalized $\mathrm{CB}[6]$ derivatives by the reaction with substituted phthalaldehydes, have built on this work and published the preparation of phenol-substituted CB[6] (14), its transformation into propargyloxy compound (15). Cycloaddition of (15) in the presence of tris(triazolyl)methanolCu(I) (Pericás' catalyst) [12] to give stimuli-responsive triazole (16) (Scheme 3) [13]. 


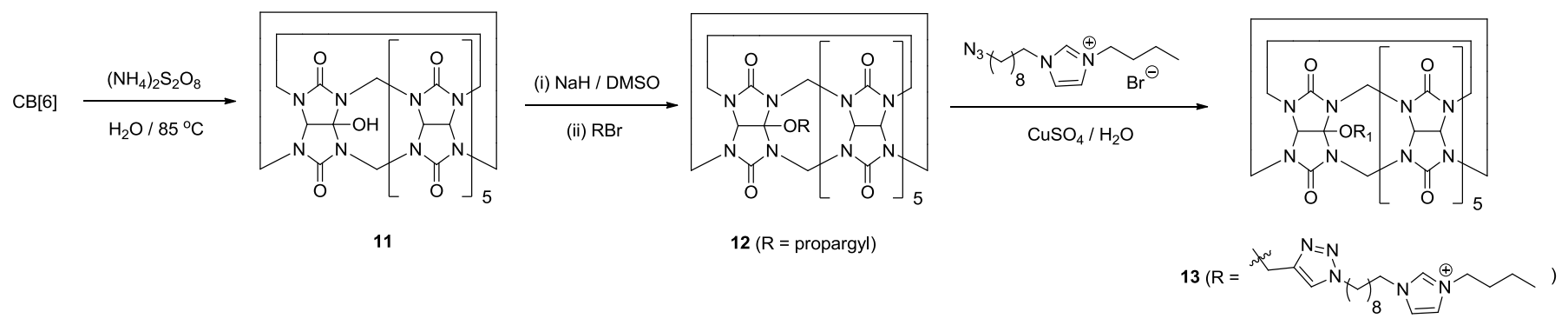

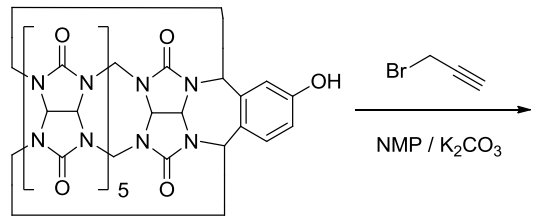

14

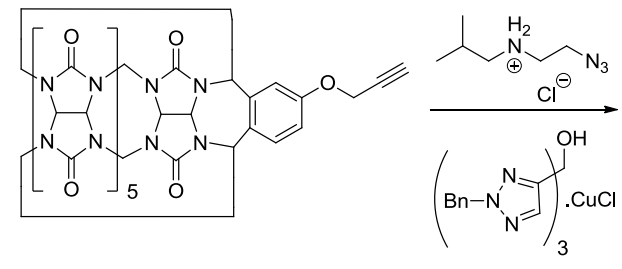

15

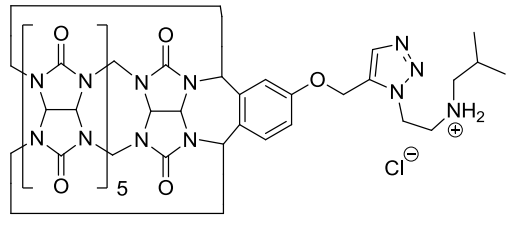

16

Miyahara and Buschmann have independently reported that acid-catalysed condensation reactions using equimolar amounts of ethyleneureas $(\mathbf{1 8}, \mathrm{R}=\mathrm{H}$ ) with formaldehyde in $4 \mathrm{~N}$ hydrochloric acid (at room temperature) provides access to hemicucurbit[n]urils (Scheme 4 , Method A, $n=6$ ) in $94 \%$ yield $[1,14,15]$. Hemicucurbit[ $n$ ] urils are cyclic and structurally-related to cucurbit[n]urils where the former are bisected along their equators as demonstrated by their X-ray structures which feature an alternate (or "zigzag") arrangement of the six ethylene urea units and a chloride ion (which is believed to act as a templating agent during the cyclocondensation process) present within the cavity. The larger macrocycle, $\mathrm{HmCB}[12]$, can be obtained in excellent yield and purity by heating an equimolar mixture of $(18, \mathrm{R}=\mathrm{H})$ and $37 \%$ formaldehyde in $1 \mathrm{~N} \mathrm{HCl}$ at $55{ }^{\circ} \mathrm{C}$ for three hours (Scheme 4, Method $\mathrm{B}, n=12,93 \%$ yield) and subsequent purification by recrystallization with chloroform [14]. The X-ray crystal structure of $18(R=H)$ has been determined and indicates cyclic structural architecture exhibiting an analogous "zigzag" conformation as the hexamer with several chloroform molecules in its crystal lattice. In contrast to $\mathrm{CB}[n] \mathrm{s}$, hemicucurbit[n]urils are soluble in non-polar solvents and exhibit significantly different behaviour in terms of their ability to selectively complex to small molecules (e.g. formamide, $\mathrm{CHCl}_{3}$ and propargyl alcohol), anions (e.g. $\mathrm{Cl}^{-}, \mathrm{SCN}^{-}, \mathrm{Br}^{-}$and I-) and cations $\left(\mathrm{Co}^{2+}, \mathrm{Ni}^{2+}\right.$ and $\left.\mathrm{UO}_{2}{ }^{2+}\right)$ but not alkali or alkaline earth metals, $\mathrm{Ag}^{+}$or $\mathrm{NH}_{4}{ }^{+}$ions $[1,3,5,14,15]$. 


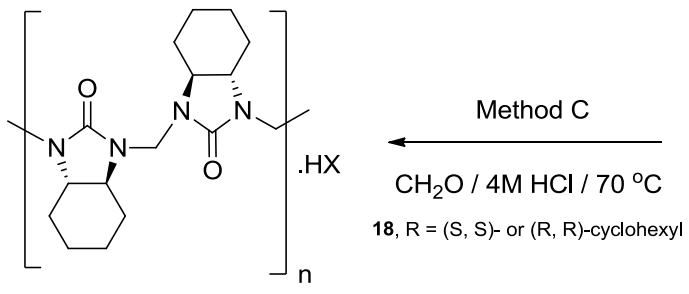

17

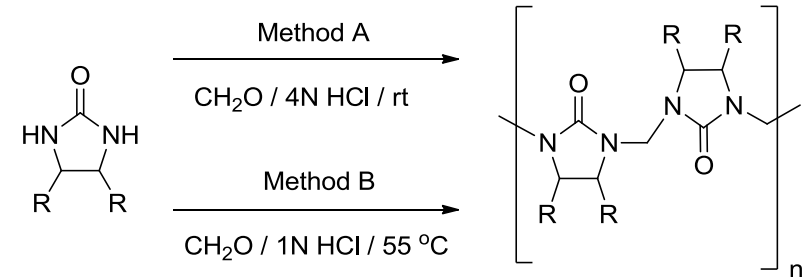

18

19

Scheme 4. Synthesis of hemicucurbit $[n]$ urils and chiral hemicucurbit $[n]$ urils

Recently Aav et al. have disclosed the chiral $(R, R)$-cyclohexylhemicucurbit[6]uril and $(S, S)$ cyclohexylhemicucurbit[6]urils (17), prepared (as their corresponding hydrochloride and hydrobromide salts) from equimolar amounts of the enantiopure perhydrobenzimidazolines $(\mathbf{1 8}, \mathrm{R}=(S, S)$ - or $(R, R)$-cyclohexyl) and paraformaldehyde in $4 \mathrm{M}$ hydrochloric (or hydrobromic) acid at $70{ }^{\circ} \mathrm{C}$ in $75-85 \%$ yield after flash chromatography (Scheme 4, Method C). The enantiopure cyclohexylhemicucurbit[6]urils formed 1:1 complexes with $\mathrm{Cl}^{-}, \mathrm{Br}^{-}$, carboxylic and thiolacetic acids and amines. Diastereomeric complexes with $(R)$ - and $(S)$-O-methylmandelic acid in organic media were also prepared [16].

Bambus[n]urils (BU[n]s), which combined the structural features of both cucurbit[n]urils and hemicucurbit[n]urils, were first disclosed by Sindelar et al. in 2010 and subsequently patented in 2011 [8, 17]. The cyclic hexamer, $\mathrm{Me}_{12} \mathrm{BU}$ [6] (20, $\mathrm{R}=\mathrm{Me}$, as the $\mathrm{HCl}$ salt) was prepared as a white powder, in $30 \%$ yield, by heating $2,4-$ dimethylglycoluril $(\mathbf{2 1}, \mathrm{R}=\mathrm{Me}$ ) and paraformaldehyde in $5.4 \mathrm{M} \mathrm{HCl}$ for $24 \mathrm{~h}$ (Scheme 5 , Method A). The structure of $\mathrm{BU}[6]$ was confirmed by X-ray analysis of microcrystals (formed by slow evaporation of $\mathrm{BU}[6]$ in $\mathrm{EtOH}-\mathrm{CHCl}_{3}$ tetrabutylammonium chloride) and indicated a cyclic structure exhibiting a hyperbolic conformation (3, Figure 1) with the chloride anion situated within the $6.4 \AA$ cavity and the tetrabutylammonium cation located outside the macrocycle. 


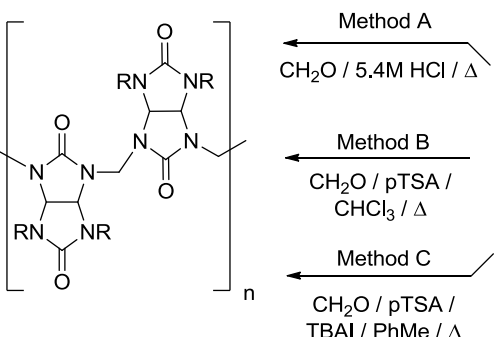

20

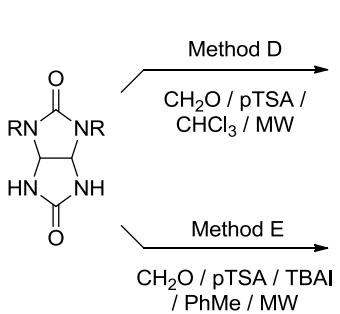

21

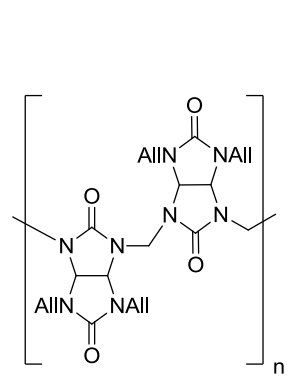

22

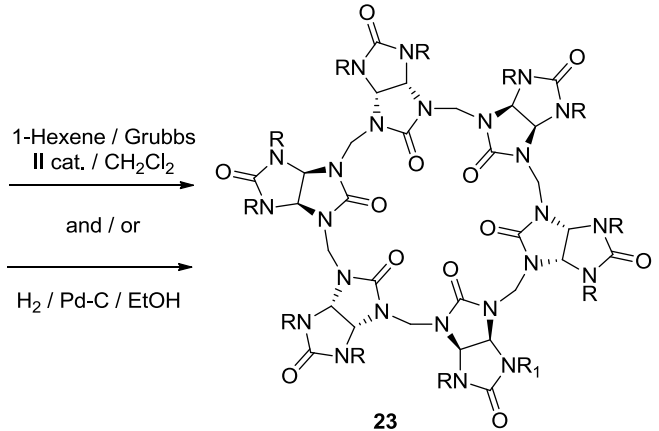

Scheme 5. Synthesis of bambus[ $n]$ urils $(n=4,6,12)$. Method A (reflux, 24h); Method B (reflux, 24h); Method C (reflux, 22h); Method D (200W, $\left.75^{\circ} \mathrm{C}, 72 \mathrm{~h}\right) ;$ Method E (200W, $\left.110{ }^{\circ} \mathrm{C}, 4 \mathrm{~h}\right)$

Subsequent to this report a number of bambus[n]urils were synthesised, from their prerequisite 2,4-disubstitutedglycoluril (20, $\mathrm{R}=\mathrm{Pr}, \mathrm{Bn}$ ) derivatives [18], using either: (i) an equimolar mixture of paraformaldehyde and $p$ toluenesulfonic acid (pTSA) in refluxing chloroform (Scheme 5, Method B) or (ii) an equimolar mixture of paraformaldehyde, $p$-toluenesulfonic acid and tetrabutylammonium iodide (TBAI) in refluxing toluene (Scheme 5, Method C). Anion-free bambus[n]urils were originally prepared by sequential displacement of the chloride anion within the cavity with a suitable solvent (e.g. MeCN), however, more robust methods involving displacement of the chloride anion with an iodide ion and the concomitant removal via oxidation (with hydrogen peroxide in the absence of light; $92 \%$ yield) or photocatalytically (using flash photolysis and titanium dioxide; $84 \%$ yield) have been recently reported [19].

Bambus[ $n]$ urils exhibit solubility in both organic and aqueous media and are primarily capable of forming stable complexes with various anions (e.g. $\mathrm{Cl}^{-}, \mathrm{Br}^{-}, \mathrm{I}^{-}, \mathrm{BF}_{4}^{-}, \mathrm{NO}_{3}{ }^{-}$and $\mathrm{CN}^{-}$) with significant selectivity [8, $\left.17-19\right]$. A number of theoretical studies of the affinity of $\mathrm{BU}[6]$ with various cations (e.g. $\mathrm{H}^{+}, \mathrm{Cs}^{+}$) and anions (e.g. $\left.\mathrm{F}^{-}, \mathrm{CN}^{-}\right)$using quantum mechanical density functional theory $[20,21]$ have also been reported.

The application of microwave-mediated synthetic chemistry has grown in significance over the last two decades and it is now routinely used in the chemical and pharmaceutical industries for parallel and combinatorial synthesis. Since microwave energy (operating at a frequency of $2.45 \mathrm{GHz}$ ) can be focussed directly into the reactive components [22, 23], rather than being transferred from the bulk solvent by convection/conduction (as occurs in regular hotplate/oil bath reactions), microwave irradiation is able to greatly enhance the rate and overall yield of many reactions [22]. It is common for microwave-assisted processes to be significantly faster than 'conventional' methods; taking minutes 
instead of days and/or seconds instead of hours [22, 23]. Microwave-mediated processes are, therefore, more economic in terms of overall energy efficiency, but also because fewer side-products are (in general) formed during these processes leading to simple work-up and purification of the desired targets [22, 23].

The application of microwave-assisted synthesis for the production of cucurbit[n]urils (Scheme 1 , Method C) was first patented by Kim et al. using an equimolar mixture of $(5, R=H)$ and paraformaldehyde in $9 \mathrm{M}$ sulphuric acid. Microwave irradiation for 45 s gave a mixture of $\mathrm{CB}[5](15 \%), \mathrm{CB}[6](45 \%), \mathrm{CB}[7](20 \%)$ and $\mathrm{CB}[8](15 \%)$ respectively [24]. Sutcliffe and Wheate reinvestigated and optimised the application of microwave-assisted synthesis in the production of $\mathrm{CB}[\mathrm{n}] \mathrm{s}$ and by varying the reaction time $\left(1-10\right.$ mins), temperature $\left(100-200^{\circ} \mathrm{C}\right)$ and the type of acid used to promote the cyclocondensation reaction. The study indicated that after irradiation $\left(160^{\circ} \mathrm{C}\right)$ a mixture of $\mathrm{CB}[\mathrm{n}] \mathrm{s}$ was produced using either 12M hydrochloric (Scheme 6, Method B) or 98\% sulfuric acids (Scheme 6, Method C) [25]. Hydrochloric acid, after 10 minutes, was found to produce a mixture of $\mathrm{CB}[5]$ (23\%), $\mathrm{CB}[6](58 \%), \mathrm{CB}[7]$ (13\%) and $\mathrm{CB}[8](6 \%)$ which approximated the distribution reported by Kim (Scheme 6, Method A), whilst sulfuric acid, after 3 minutes, produced mostly $\mathrm{CB}[6]$ (circa. 90\%) with small amounts of $\mathrm{CB}[5]$ and $\mathrm{CB}[7]$.

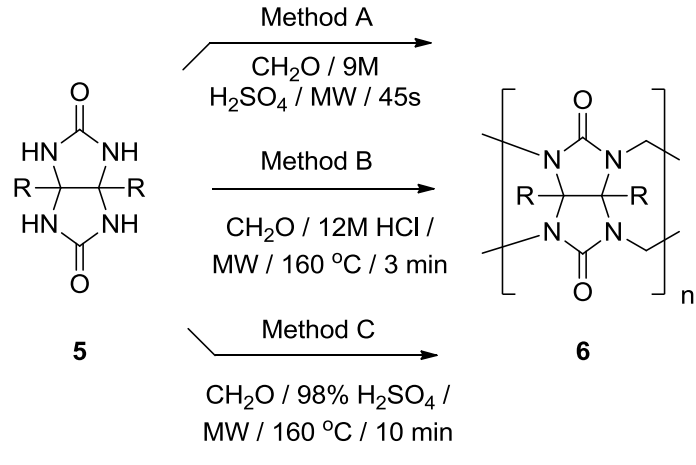

Scheme 6. Microwave-assisted synthesis of cucurbit $[n]$ urils $(n=5,6,7,8$ or 10$)$.

The type of the acid used was a critical factor as phosphoric, formic, acetic and trifluoroacetic (TFA) acids failed to produce any of the desired $\mathrm{CB}[n] \mathrm{s}$ and nitric acid was unsuitable due to a violent off-gassing that occurred during dissolution of the starting materials. Mixtures of strong and weak acids (e.g. $\mathrm{H}_{2} \mathrm{SO}_{4}$ :TFA in ratios between 20:80 and $80: 20)$ produce $C B[n]$ less efficiently and with $C B[6]$ as the prominent macrocycle. Scale-up of the reaction by $2-4 x$ was shown to be viable with the distribution of the $\mathrm{CB}[n]$ s produced remaining unchanged. 
Though the application of microwave-assisted synthesis has not, to date, been applied to the preparation of hemicucurbit[n]urils, Sutcliffe and Wheate's method for cucurbit[n]urils has been successfully applied to the synthesis of novel bambus[n]urils by Heck et al. (Scheme 5, Methods D and E) [26]. Irradiation of an equimolar mixture of 2,4-allylglycoluril (21, $\mathrm{R}=$ Allyl), paraformaldehyde and catalytic $p$-toluenesulfonic acid in chloroform (75 ${ }^{\circ} \mathrm{C}, 72 \mathrm{~h}$, Scheme 5, Method D) afforded ally| $\mathrm{I}_{8} \mathrm{BU}[4](23, n=4,85 \%$ yield) and irradiation of an equimolar mixture of paraformaldehyde, $p$-TSA and TBAl in toluene $\left(110^{\circ} \mathrm{C}\right.$, $4 \mathrm{~h}$, Scheme 5 , Method E) gave the hexamer, ally ${ }_{12} \mathrm{BU}[6](\mathbf{2 3}, n$ $=6, R=R_{1}=$ allyl), in $60 \%$ yield - which was vastly superior to the classical route (circa. $20 \%$ yield). Subsequent manipulation of ally| ${ }_{8} \mathrm{BU}[4]\left(23, n=4, \mathrm{R}=\mathrm{R}_{1}=\right.$ allyl) was achieved through cross-metathesis with Hovyeda-Grubbs catalyst (90\% yield) and concomitant hydrogenation with Pd-C (100\% yield) providing access to three further examples of this emerging class of macrocycles: $\operatorname{propen}_{8} \mathrm{BU}[4]\left(\mathbf{2 3}, n=4, \mathrm{R}=\mathrm{R}_{1}=\mathrm{CH}=\mathrm{CH}_{-} \mathrm{CH}_{3}\right)$; allyl ${ }_{7} \mathrm{HepBU}[4](23, n=$ 4, $\mathrm{R}=$ allyl, $\left.\mathrm{R}_{1}=\mathrm{CH}_{2}-\mathrm{CH}=\mathrm{CH}-\left(\mathrm{CH}_{2}\right)_{3} \mathrm{CH}_{3}\right)$ and $\operatorname{Pr}_{8} \mathrm{BU}[4]\left(23, n=4, \mathrm{R}=\mathrm{R}_{1}=\right.$ propyl).

\section{Applications}

The high binding affinity and selectivity of cucurbit $[n]$ urils has attracted significant attention, as such, $\mathrm{CB}[n] \mathrm{s}$ have demonstrated enormous potential in a range of applications, including microelectronics and nanomachine components, chromatography and waste remediation, drug delivery, biosensors, and self-assembly and reaction catalysis [1 -7]. The construction of self-assembling molecular machines, which can switch between two different states via a chemical, photochemical or electrochemical stimulus, is currently of interest and the $\mathrm{CB}[n]$ family is ideally suited for such applications due to their recognition properties. A detailed discussion of these applications is outside the scope of this chapter, so we recommend the extensive reviews by Lagona et al. [1], Kim [3] and Isaacs [4]. Functionalised cucurbit[n]urils have also used for a number biomimetic applications as artificial biological structures and processes in living cells such as: selective ion channels, vesicles for targeted drug delivery and even gene transfection $[1,3,5,7]$. Hemicucurbit[n]urils have limited applications but have been utilised as supramolecular catalysts in the esterification of 4-methoxy-4-oxobut-2-enoic, acrylic and benzoic acids using methanol [27] and patented as potential cosmetic agents [28]. In contrast to the cucurbit[n]uril and hemicucurbit[n]uril families, the detailed recognition properties and practical applications of bambus[ $n]$ urils remain to be explored.

\section{Future Perspective}

Many of the early concerns with the cucurbit[n]uril family, such as poor solubility, have been circumvented, either by the use of salts or by chemical modification of the macrocycle itself. Since the turn of the century, the scientific literature has expanded greatly, aided by the commercial availability of many of the $C B[n] s$ and new synthetic procedures for the functionalization of these materials, which has widened their use greatly. The utility of $\mathrm{CB}[n] \mathrm{s}$ in an assortment of chemical (microelectronics, chromatography, waste remediation and self-assembly or reaction 
catalysts) and pharmaceutical (nanomachines, drug delivery and biosensors) applications have been consistently demonstrated in vitro and in a few in vivo examples. Their progress from "bench to bedside", however, is reliant on the future development of functionalized $\mathrm{CB}[n] \mathrm{s}$ and reproducibility of structurally consistent $\mathrm{CB}[n] \mathrm{s}$ and their hostguest complexes. Cucurbit[n]urils have already been revealed to prevent the degradation of some drugs and diminish their toxicity. The development of new $\mathrm{CB}[n] \mathrm{s}$ with suitable functional groups (e.g. amine and carboxylic acids) will allow the growth of targeted drug-delivery vehicles through the attachment of biologically relevant substrates, antibodies, peptides or aptamers to the $\mathrm{CB}[n]$ s. To date, few biologically useful functionalized $\mathrm{CB}[n] s$ have been reported and the application of novel and efficient synthetic methodologies (for example: microwave-assisted organic synthesis [MAOI]) may provide instrumental in the expansion of the $\mathrm{CB}[n] \mathrm{s}$ family.

A requirement of pharmaceutical drug approval is to demonstrate the ability to produce new drugs (or, in this case, $\mathrm{CB}[n]$-based drug-delivery systems) in a single form both robustly and reproducibly. It is well documented that $\mathrm{CB}[n] \mathrm{s}$ produce a range of different crystal polymorphs (i.e. the ability of a solid material to exist in more than one crystalline form) based on $\mathrm{CB}[n]$-drug interactions and the crystal's hydration state [25]. As such, the crystallization processes of $\mathrm{CB}[n] \mathrm{s}$ and their host-guest complexes needs to be scrutinized in great detail, in order to determine the number of different polymorphs which may form, the physicochemical processes that drive their crystallization and solid-state packing, as well as methods to produce $\mathrm{CB}[n]$-drug formulations suitable for clinical use (e.g., oral, liquid or solid dosage forms). Though the application of hemicucurbit[ $n]$ urils and bambus[ $n]$ urils has, to-date, not been exploited as much as their cucurbit[n]uril cousins their potential in biological, pharmaceutical and chemical science, especially in light of new derivatives being reported in the literature, is far from exhausted.

\section{Executive Summary}

Cucurbit $[n]$ urils $(\mathrm{CB}[n] \mathrm{s})$ are macrocycles made from the acid-catalyzed condensation reaction of glycoluril and formaldehyde.

Hemicucurbit[n]urils (HemCB[n]s) are macrocycles made from the acid-catalyzed condensation reaction of ethyleneureas and formaldehyde.

- $\quad$ Bambus[n]urils (BU[n]s) are macrocycles made from the acid-catalyzed condensation reaction of 2,4disubstituted glycoluril subunits and formaldehyde.

$\mathrm{CB}[n] \mathrm{s}$ have applications in many chemical, pharmaceutical and biomimetic fields, however, the application hemCB $[n] s$ and $\mathrm{BU}[n] s$ have, to-date, not been exploited to their full potential.

Microwave synthesis can applied as an efficient and low cost method on the industrial scale for the production of $\mathrm{CB}[n] \mathrm{s}$ and $\mathrm{BU}[n] \mathrm{s}$ for a variety of applications. 
[1] Lagona J, Mukhopadhyay P, Chakrabarti S, Isaacs L. The cucurbit[n]uril family. Angew. Chem. Int. Ed. Engl., 44 (31), 4844-4870 (2005).

[2] Kim K, Selvapalam N, Ko YH, Park KM, Kim D, Kim J. Functionalized cucurbit[n]urils and their applications. Chem. Soc. Rev., 32 (2), 267-279 (2007).

[3] Huang W-H, Liu S, Isaacs L. Cucurbit[n]urils, in: Modern Supramolecular Chemistry: Strategies for Macrocycle Syntheis, ed. Diederich F, Stang PJ, Tykwinski RR. Wiley-VCH Verlag GmbH \& Co., Weinheim, 113-142 (2008).

[4] Isaacs L. Cucurbit[n]urils: from mechanism to structure and function. ChemCommun., 6, 619-629 (2009).

[5] Davis F, Higson S. Cucurbit[n]urils, in: Macrocycles: Construction, Chemistry and Nanotechnology Applications, John Wiley \& Sons Limited, Chichester, 325-380 (2011).

[6] Masson E, Ling X, Joseph R, Kyeremeh-Mensah L, Lu X. Cucurbit[n]uril chemistry: a tale of supramolecular success. RSC Adv., 2, 1213-1247 (2012).

[7] 16 Consecutive Reviews in Isr. J. Chem., 51, 487-678 (2011).

[8] Svec J, Necas M, Sindelar V. Bambus[6]uril. Angew.Chem. Int. Ed. Engl., 49 (13), 2428-2431 (2010).

[9] Day A, Arnold AP, Blanch RJ, Snushall B. Controlling factors in the synthesis of cucurbituril and its homologues. J. Org. Chem., 66 (24), 8094-8100 (2001).

[10] Jon SY, Selvapalam N, Oh DH, Kang J-K, Kim S-Y, Jeon YJ, Lee JW, Kim K. Facile synthesis of cucurbit[n]uril derivatives via direct functionalization: Expanding utilization of curcurbit[n]uril. J. Am. Chem. Soc., 125 (34), 10186-10187 (2003).

[11] Zhao N, Lloyd GO, Scherman OA. Monofunctionalized cucurbit[6]uril synthesis using imidazolium host-guest complexation. Chem. Commun., 48 (28), 3070-3072 (2012).

[12] Ozçubukçu S, Ozkal E, Jimeno C, Pericàs MA. A highly active catalyst for Huisgen 1,3-dipolar cycloadditions based on the tris(triazolyl)methanol-Cu(I) structure. Org. Lett., 11 (20), 4680-4683 (2009).

[13] Cao L, Isaacs L. Daisy chain assembly formed from a curcubit[6]uril derivative. Org Lett., 14 (12), 2072-3075 (2012).

[14] Miyahara Y, Goto K, Oka M, Inazu T. Remarkably facile ring-size control in macrocyclizations: Synthesis of hemicucurbit[6]uril and hemicucurbit[12]uril. Angew.Chem. Int. Ed. Engl., 43 (38), 5019-5022 (2004). 
[15] Buschmann H-J, Zielesny A, Schollmeyer E. Hemicucurbit[6]uril a macrocyclic ligand with unusual complexing properties. Journal of Inclusion Phenomena and Macrocyclic Chemistry, 54 (3 - 4), 181-185 (2006).

[16] Aav R, Shmatova E, Reile I, Borissova, M, Topic F, Rissanen K. New chiral cyclohexylhemicucurbit[6]uril. Org. Lett., 15 (14), 3786-3789 (2013).

[17] Sindelar V, Svec J, Havel V. Macrocyclic derivatives of glycolurils and their preparation and use. WO2011057590 (2011).

[18] Havel V, Svec J, Wimmerova M, Dusek M, Pojarova, M and Sindelar V. Bambus[n]urils: a new family of macrocyclic anion receptors. Org. Lett., 13 (15), 4000-4003 (2011).

[19] Svec J, Dusek M, Fejfarova K, Stacko P, Klan P, Kaifer AE, Li W, Hudeckova E, Sindelar V. Anion-free bambus[6]uril and its supramolecular properties. Chem. Eur. J., 17 (20), 5605-5612 (2011).

[20] Toman P, Makrlik E, Vanura P. Theoretical study on the complexation of bambus[6]uril with cyanate and thiocyanate anions. Monatshefte fur Chemie, 143 (7), 985-988 (2012).

[21] Toman P, Makrlik E, Vanura P. Theoretical study on the protonation of bambus[6]uril. Monatshefte fur Chemie, 143 (3), 373-376 (2012).

[22] Kappe CO. Microwave dielectric heating in synthetic organic chemistry. Chem. Soc. Rev., 37, 1127-1139 (2008).

[23] Kappe CO. Controlled microwave heating in modern organic synthesis. Angew.Chem. Int. Ed. Engl. 43 (46), 6250-6284 (2004).

[24] Kim K, Samal S, Kumar NR, Selvapalem N, Oh DH. Process of preparing glycolurils and cucurbiturils using microwave. US2007/0232809 (2007).

[25] Wheate NJ, Patel N, Sutcliffe OB. Microwave synthesis of cucurbit[n]urils. Future Med. Chem., 2, 231-236 (2010).

[26] Rivollier J, Thuery P, Heck M-P. Extension of the bambus[n]uril family: Microwave synthesis and reactivity of allylbambus[ $n$ ]urils. Org Lett, 15 (3), 480-483 (2013).

[27] Cong H, Yamato T, Feng X, Zhu T. Supramolecular catalysis of esterification by hemicucurbiturils under mild conditions. Journal of Molecular Catalysis A: Chemical, 365, 181-185 (2012).

[28] Hoeffkes $H$, Knuebel G, Giesa H. Cosmetic agents containing macrocycles selected from the group of cucurbituril and hemicucurbituril derivatives. DE102006011317 (2007). 